\title{
Research on Man-Machine Interface Design in Intelligent Terminal Products
}

\author{
$\mathrm{Ze} \mathrm{Fu}{ }^{\mathrm{a},{ }^{*}}$ \\ Yiwu Industrial and Commercial College, Yiwu, 100049, China. \\ a, *447472145@qq.com
}

\begin{abstract}
We know that good design is to give people a good experience. In recent years, there have been various kinds of intelligent terminal, its function is varied and its operation is low, so it is suitable for the use of most people. Moreover, as a new technology product, it undoubtedly has a great influence on transportation mode and information dissemination. More importantly, compared to the traditional terminal equipment, the design of the human computer interface is very humanized, and it is also one of the reasons for its popularity all over the world. These intelligent products are excellent in all aspects, and their performance is very satisfactory. The satisfaction degree is no less than the comfort, satisfaction, convenience and superiority of high quality artificial service. The original intention of intelligent products is multiple, but one thing is common, that is to say its original purpose is to make people gradually realize the convenience brought by the industrial revolution. We often say to improve the sense of experience of the people, to improve the sense of satisfaction of the people, to improve the sense of gain of the people, and to improve the sense of existence and happiness of the people in the present and contemporary developed society. The feeling of experience is a very mysterious word. People live in a society, and we are all one of them. Social relationship is a network connecting these very many points. We live happily on this website. Our social relationship with others is a line connecting these nodes. The premise that our society can work in an orderly way is that the net should be dense and compact. If each of us has a sense of experience and a good sense of experience, then this society can operate efficiently, and in turn, we can get more experience, which makes us more experienced and enrich our life. In this paper, the design of human computer interface in intelligent terminal is studied. By introducing the various kinds of intelligent terminals, the principle of human-machine interface is analyzed in detail. The goal of human-machine balance is to find a guide for people in the design of human-computer interaction interface. The survey shows that this combination is very promising and is what the general public wants.
\end{abstract}

Keywords: Human-Computer Interaction; Interface Design; Visual Design; Intelligent Terminal; User Experience.

\section{智能终端产品人机界面设计研究}

\author{
傅泽 \\ 义乌工商职业技术学院, 义乌, 100049, 中国
}

我们知道好的设计是给人们一个好的体验。近年来, 出现了各种各样的智能终端, 其功能多 样, 操作性低, 适合大多数人的使用。此外, 作为一种新的技术产品, 它无疑对运输方式和 信息传播有很大的影响。更重要的是, 与传统的终端设备相比, 人机界面的设计是非常人性 化的, 也是全球普及的原因之一。这些智能产品在各个方面都是优秀的, 性能非常令人满意。 满意程度不低于优质人工服务的舒适性、满意度、便利性和优越性。智能产品的初衣是多方 面的, 但有一点是共同的, 即其最初的目的是使人们逐渐认识到工业革命带来的便利。我们 经常说, 要提高人民的经验意识, 提高人民的满足感, 提高人民的利益, 提高当代和当代发 达国家人民的生存和幸福感。体验的感觉是一个非常神秘的词。人们生活在一个社会里, 我 们都是其中之一。社会关系是连接这些许多点的网络。我们幸福地生活在这个网站上。我们 
与他人的社会关系是连接这些节点的一条线。我们的社会有秩序地工作的前提是网络应该是 密集而紧凑的。如果我们每个人都有一种经验感和良好的经验感, 那么这个社会可以有效地 运作, 反过来, 我们可以获得更多的经验, 这使我们更有经验, 丰富我们的生活。本文研究 了智能终端中人机界面的设计。通过对各种智能终端的介绍, 详细分析了人机界面的工作原 理。人机交互的目标是在人机交互界面的设计中寻找人的引导。调查表明, 这种结合是非常 有前途的, 也是大众所希望的。

\section{关键词: 人机交互; 界面设计; 视觉设计; 智能终端; 用户体验.}

\section{1. 引言}

近年来越来越多产品的设计都在向着智能化的方向高速发展, 由此给我们带来了一系列的智 能终端 [7]。比如智能穿戴设备: 智能手环, 可以简单且舒适的佩戴在人们的手上, 它在帮 助人们监测一些自身生命特征的同时，还可以利用某些诸如蓝牙和红外的技术对这些特征的 数据进行上传以供分析 [2]; 智能手机：它打破了传统手机功能单一的枷锁 [8]。多线程的任 务操作方式 [3], 简洁的界面设计, 良好的人机交互体验 [4], 都给人们以不俗的使用体验和 视觉享受。使用这些智能产品不用我们过多的思考, 这些产品就会根据我们的使用习惯, 记 录下来并给我们的行为予以指导 [9]。现阶段智能终端的例子还有很多，这里不再一一赘述。 我们常说, 智能终端的出现改变了人们的生活方式 [10]。传统的非智能的设备的使用给人们 带来了不是很好的使用体验, 它们操作复杂, 且不易携带。比如鼠标和键盘, 它们带给我们 了不同的世界 [5], 但是他们是非智能的, 多多少少都会给我们一种冷漠感 [11]。而智能产品 在这方面的优势是不言而喻的，对于它们的交互过程类似于人与人之间的交流过程，使人倍 感舒适，无需专业的技巧，无需专业的培训我们即可操控它。而且，这些智能设备的发明无 论从时间还是空间上都给我们以强烈的真实感 [6]。它们有丰富的输入和输出技术, 有着近乎 完美的硬件支持，有着数量庞大的受众 [12]。目前，有很多公司都已经有先见之明的不惜花 费大量资金和科研人员投入到智能终端设备的研发中。他们的工作无不在一定程度上推动了 社会的进步，推动了智能终端设备的长足发展。这些公司遍布全世界，比如华为公司、中兴 公司、小米公司、苹果公司，他们的研究都是跨时代的。

本文里, 我们基于用户体验感的角度出发, 详细的说明了人机交互的界面设计在智能终端的 研发中起到的主导作用。这些提议以及方案设计对于智能设备来说都是比较有指导意义的, 下面我们来分门别类的介绍这些理念, 也在最后给出我们的设计方案, 相信对于智能家居的 工程应用来说其是个强有力的推动。

\section{2. 智能终端}

近些年来，随着计算机技术的不断发展，人们为寻求更自然的沟通渠道不断努力。随着智能 手机的普及, 以智能手机作为控制平台的智能终端设备, 与最初的智能家居相比, 它的优势 是很大的, 不仅如此我们还省去了布线的麻烦 [13]。我们只需要一个简单的动作就可以使家 居显得更智能, 这正是我们朝思暮想的, 也是我们非常渴望得到的。目前, 有很多公司都已 经有先见之明的不惜花费大量资金和科研人员投入到智能家居的研发中。

我们在使用一些产品时, 不可避免的肯定在第一时间想到的是这件产品设计的安全问题, 在 这一方面，智能家居完全可以让用户放心 [14]。无论小区硬件设施以及人员管理得是多么的 到位, 我们都要有心观察安全问题, 这是非常有必要的。生活中一些紧急情况的发生也不在 少数，一旦发生了，我们就要有足够的精力和常识去应对，否则后果将不堪设想。

智能终端的服务对象就是我们一普通人民大众, 所以说, 脱离了用户体验的智能终端即使再 方便再智能, 那么它也并不是我们想要的。而且, 我们更希望它能有所创新, 因为创新是促 
进社会发展的源泉, 为社会生产力提供了不竭的动力 [15]。基于此, 我们提出了基于用户体 验感的智能终端设计的方案。以用户为核心, 为用户谋福利、谋方便。

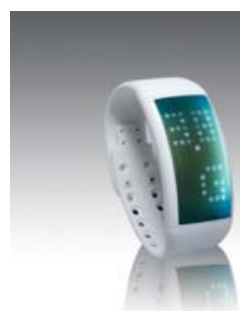

图1. 智能手环

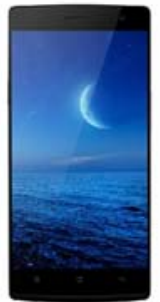

图2.智能手机

\section{3. 人机交互界面设计}

之所以智能终端设备如此的受到人类的青睐, 究其原因, 简洁高效的人机交互方式功不可没, 也就是说其视觉 UI 做的是非常好的 $[16]$ 。

所谓的人机交互可以理解为人与机器的交互, 简单的说就是指利用一系列的方法在用户或操 作人员与计算机或智能终端之间建立一定的交流机制。通常情况下，对于人机交互我们可以 作如下的简要理解。在传统的计算机中对于指令是由一系列繁杂的 0 和 1 的组合来完成的, 通过 0 和 1 的组合将我们想要表达的信息告知计算机，计算机再以某种形式将这些信息作一 定的处理, 这样就是人与计算机交流的途径。交互是对一种情境描述中如何刻画的信息传达, 而界面是实现交互的一种手段。如果要以内容和形式来区分交互和界面的关系，那么内容会 是交互, 而界面就是形式; 交互和界面的最终目的是在满足人们需求的前提下工作, 是一种 解决问题的手段 [17]。

在人机交互界面的设计中, 我们也要引入视觉 UI 的概念。也就是说我们要从从生物学的角度 出发, 通过对大小、方位以及颜色的感知来帮助我们设计更加好的, 更加人性化的产品。在 我们研究人机交互的过程中, 有一些东西是我们需要时刻牢记的 [18]。简要的归纳如下: 在 设计人机交互的过程中，应该从录取设备入手，充分考虑人机界面的问题，因为只有这样才 能彻底解决关于设备实用性的问题，才能彻底提高人机交互的速度。

要充分考虑到设计的目标不仅仅是对于计算机系统的可用性, 还要有对设计最佳的系统, 也 就是说整个系统才是我们的使用目标。从另一方面我们可以这样说, 人机交互里我们最感兴 趣的是如何让人们更好的完成工作。至于最好的人机交互过程, 这并不是我们最想要的, 也 就是说我们不能顾此失彼, 要时刻把握自己的方向。

\section{4. 基于智能终端的设计}

所谓的基于智能终端的人机交互设计，也就是基于计算机的人机交互的设计。对于我们普通 的用户来说, 我们所关心的始终是交互界面的方便性以及实用性。下面即是人机交互过程中 信息处理的流程:

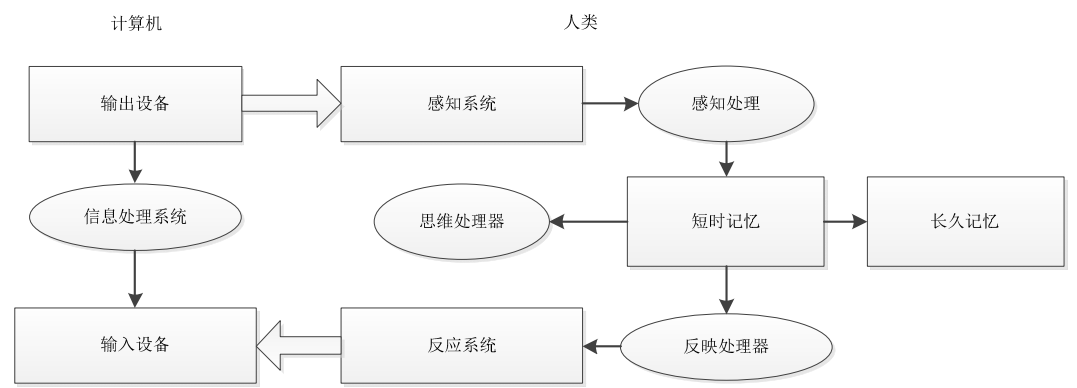

图3. 简易的人机交互过程示意图

人性化设计的目的就是尽可能的将计算机的输入和输出设备等等的一系列信息 [19], 以人类 能够感知、能够理解的方式提供给广大的受众。与此同时, 键盘鼠标和话筒这些外置的输入 
设备可以作为用户和计算机交流的桥梁。目前, 大多数的人机界面交互的设计也都是这个方 式。

\section{5. 结语}

在以知识经济和数字时代为特征的非物质的社会中, 我们设计的产品用户界面确实应该考虑 客户的个人隐私空间和自我的实现欲望。因为保护个人的隐私空间是法律所必须的，违反了 这个就是违法的, 是为大众所不容的。用户追求自我实现的愿望也逐渐的促使设计朝着非物 质的方向发展。非物质时代的前提下用户对个性张扬的渴望和对快速变化的时尚的追求, 伴 随着强烈的情感的特质就产生了非物质时代的人机交互界面设计。迄今为止, 智能终端在中 国已经历了许多年的发展, 这许多年来它无时无刻不在为我们普通大众给予服务。但是与此 同时我们也更应该注重人们体验感的提升, 因为对于一项科技来说, 如果它有悖于人们的舒 适感, 那这项科技就是没有意义的, 或者说是没有必要存在的, 是多余的。本文中, 我们重 点研究了基于人机交互界面设计的智能终端的设计方案, 它有很多便利之处。但是它绝不是 最完美的，因为最完美的设计永远在未来，我们也更将以渴望的姿态面对未来。

\section{Acknowledgements}

2017 Zhejiang Provincial Department of Education General Questions (Y201737448).

\section{References}

[1]. Xiao Hong, Guo Gem. Discussion on the Visual Design Principles of Multi-Sense Human-Computer Interface[J]. Packaging Engineering, 2012, 33 (8):35-37.

[2]. LIL Kang, Jan Xiao, LI Shi-good. Application of Interaction Feedback Mechanism in the Design of Products [J]. Packaging Engineering, 2009, 30 (11):123-125.

[3]. Hyun K H, Kim E H, Kwak Y Emotional Feature Extraction Method Based on the Concentration of Phoneme Influence for Human-Robot Interaction [J]. Advanced Robotics, 2010, 24 (1-2): 33-35.

[4]. Kim h R, Kwon D Computational Model of Emotion Generation for Human-Robot Interaction Based on the Cognitive Appraisal Theory[J]. Journal of Intelligent \& Robotics Systems, $2010,60(2): 78-80$.

[5]. Xiao Ran, LI Shi-gun, Pan Zoos-ping. The Application of Interaction Design in the Product Application of Feed-forward Mechanism [J]. Packaging Engineering, 2010, 31(18):31-34.

[6]. Zhang You-week. Human-computer Nature Interaction [J]. Défense Industry Daily, 2009 (4): 12-14.

[7]. Liu Sham. Design Psychology [M]. Shanghai: Shanghai People's Fine Arts Publishing House, 2009.

[8]. Beale R, Creed Affective Interaction: How Emotional Agents Affect Users [J]. International Journal Human Computer Studies, 2009, 67(9):121-123.

[9]. Amble, T. The social psychology of creativity's componential conceptualization, Journal of Personality and Social Psychology [M]. 1983, T 45:357-376.

[10]. De Silva R, Dutra M J, et al. Personal identity module using psych evolutionary emotion theory for social robots [J]. 2012 IEEE ROMAN: The 21st IEEE International Symposium on Robot and Human Interactive Communication. September 9-13, 2012. Paris, France: 551-557. 
[11]. BEALE R, CREED Affective Interaction: How Emotional Agents Affect Users [J]. International Journal Human Computer Studies, 2009, 67(9):121-123.

[12]. Lanfranc M, Le Gars D. Robotic implantation of deep infra-operative flat-panel CT [J]. Act Neurotic (alien). 2012 brain stimulation leads assisted by, 154 (11):2069-2074.

[13]. Amble, T. The social psychology of creativity's componential conceptualization, Journal of Personality and Social Psychology [M]. 1983, T 45:357-376.

[14]. DE Silva R, Dutra M J, et al. Personal identity module using psych evolutionary emotion theory for social robots [J]. 2012 IEEE ROMAN: The 21st IEEE International Symposium on Robot and Human Interactive Communication. September 9-13, 2012. Paris, France: 551-557.

[15]. Van Linen P H G, Wijngaards N J E, Brazier F M T. towards designing creative artificial systems, Artificial Intelligence for Engineering Design, Analysis and Manufacturing [J]. 2004, 18(3):217-225.

[16]. DE Silva R, Dutra M J, et al. Personal identity module using psych evolutionary emotion theory for social robots [J]. 2012 IEEE ROMAN: The 21st IEEE International Symposium on Robot and Human Interactive Communication. September 9-13, 2012. Paris, France: 551-557.

[17]. HYUN K H, KIM E H, KWAK Y Emotional Feature Extraction Method Based on the Concentration of Phoneme Influence for Human-Robot Interaction[J]. Advanced Robotics, 2010, 24(1-2):33-35.

[18]. Van Linen P H G, Wijngaards N J E, Brazier F M T. towards designing creative artificial systems, Artificial Intelligence for Engineering Design, Analysis and Manufacturing [J]. 2004, 18(3):217-225.

[19]. Amble, T. The social psychology of creativity's componential conceptualization, Journal of Personality and Social Psychology [M]. 1983, T 45:357-376. 